\title{
An Innovative Approach to Track Moving Object based on RFID and Laser Ranging Information
}

\author{
Gaoli Liang ${ }^{1}$, Ran Liu ${ }^{1,2 *}$, Yulu Fu ${ }^{1}$, Hua Zhang ${ }^{1}$, Heng Wang ${ }^{1}$, Shafiq ur Rehman ${ }^{1,3}$, \\ Mingming Guo ${ }^{1}$ \\ ${ }^{1}$ School of Information Engineering, Southwest University of Science and Technology, China \\ [e-mail: m18381669557@163.com] \\ ${ }^{2}$ Engineering Product Development, Singapore University of Technology and Design, Singapore \\ [e-mail: ran_liu@sutd.edu.sg] \\ ${ }^{3}$ Department of Computer Science, Lasbela University of Agriculture, Water and Marine Sciences, Pakistan \\ [e-mail: shafiq@luawms.edu.pk] \\ *Corresponding author: Ran Liu
}

Received May 26, 2019; revised July 22, 2019; accepted July 28, 2019; published January 31, 2020

\begin{abstract}
RFID (Radio Frequency Identification) identifies a specific object by radio signals. As the tag provides a unique ID for the purpose of identification, RFID technology effectively solves the ambiguity and occlusion problem that challenges the laser or camera-based approach. This paper proposes an approach to track a moving object based on the integration of RFID and laser ranging information using a particle filter. To be precise, we split laser scan points into different clusters which contain the potential moving objects and calculate the radial velocity of each cluster. The velocity information is compared with the radial velocity estimated from RFID phase difference. In order to achieve the positioning of the moving object, we select a number of $K$ best matching clusters to update the weights of the particle filter. To further improve the positioning accuracy, we incorporate RFID signal strength information into the particle filter using a pre-trained sensor model. The proposed approach is tested on a SCITOS service robot under different types of tags and various human velocities. The results show that fusion of signal strength and laser ranging information has significantly increased the positioning accuracy when compared to radial velocity matching-based or signal strength-based approaches. The proposed approach provides a solution for human machine interaction and object tracking, which has potential applications in many fields for example supermarkets, libraries, shopping malls, and exhibitions.
\end{abstract}

Keywords: laser clustering, particle filtering, received signal strength (RSS), RFID phase, velocity matching

This research is supported by the National Natural Science Foundation of China (61601381, 61701421, and 61471306), the Sichuan Science and Technology Program (2019YFH0161), and the China's 13th Five-Year Plan in the Development of Nuclear Energy (2016[1295]). 


\section{Introduction}

At present, indoor positioning has drawn more and more attention thanks to the rapid development of location-based services (LBS), such as city guidance, car parking, and asset tracking [1][2]. The most famous positioning system is GPS (Global Positioning System), which has been widely used in many areas [2], but GPS is not suitable for indoor environments due to the occlusion of signals from buildings. Indoor positioning is assumed to be more challenging [2][3], as it needs to consider the indoor multipath effects, uneven signal strength distribution, and signal interference and fluctuations.

A typical indoor positioning system may use different technologies for positioning, for example ultra-wideband (UWB) [4], Wifi [5], and RFID [6] [7]. Recent research shows a growing interest in deploying RFID technology in industrial environments (for example access management, logistics, and retail) and people's daily life. RFID achieves identification of objects through electromagnetic waves [6]. Due to its unique and contactless features of identification, RFID gradually becomes the preferred technology for indoor positioning. For example, Liu et al. [8] [9] used RFID for the inventory of a library using a mobile robot. Xie et al. [10] used RFID to create a smart bookshelf that provides automated and intelligent book management.

In the literature, extensive researches have been done on localization based on RFID technology. Cicirelli et al. [11] studied the feasibility to use passive RFID tags for localization. They used a probabilistic sensor model to achieve the positioning of an RFID tag by taking the measurements at different positions of a moving robot. However, this method requires a manual calibration stage to construct the sensor model. Vorst et al. [12] proposed a semi-autonomous method to construct the RFID sensor model. They placed some known RFID tags in an environment, collected the data in that environment with a robot, and then built an off-line RFID sensor model. Subsequently, Joho et al. [13] used the signal strength information to establish a more accurate sensor model and improved the overall positioning accuracy. Hightower et al. [14] proposed SpotON, which uses the signal strength from at least three readers to calculate the location of the tag by triangulation. Some researches also focused on the 3D (three dimensional) positioning of RFID tags. Wu et al. [15] proposed an improved 3D LANDMARC indoor positioning method, which can reduce the positioning error.

Taking into account the limitations of pure RFID systems, some researchers fused other sensory information to reduce the positioning error of a tag. Macomber et al. [16] proposed to use visual cameras to localize LED-enhanced passive RFID tags. Rohweder et al. [17] combined the environmental structure information to reduce the positioning error of a tag. Deyle et al. [18] proposed an optimized searching algorithm that can help a service robot to quickly locate RFID tags. Some researchers also focused on dynamic objects tracking using RFID tags. Liu et al. [8] [19] combined RFID and laser sensor to follow a dynamic RFID tag with obstacle avoidance capabilities using a mobile robot. Their algorithm combines two kinematics models and a dual particle filter to achieve fast tracking of dynamic RFID tags. Fu et al. [20] studied a new algorithm that uses particle filters to fuse RFID phase and laser clustering from laser sensor to locate moving objects. Scherhaufl et al. [21] localized UHF RFID tags by evaluating the phase-of-arrival signal of a tag that is backscattered from several antennas. 
Many researchers in the literature also focus on the new design of antennas to improve the performance of RFID systems. Chen et al. [22] showed that the performance of the RFID antenna has a significant impact on the reading range and detection accuracy of the RFID system. Subsequently, they [23] proposed a broadband circularly polarized patch antenna suitable for the global UHF band 840-960 MHz. Qing et al. [24] proposed a segmented loop antenna for ultra high frequency (UHF) near field RFID applications. By changing the size and the separation of the backing metal plate, they [25] applied the RFID system to a smart-shelf. Alibakhshi-Kenari et al. [26] designed a miniaturized planar metamaterial antenna to decrease the antenna size and enlarge the bandwidth and gain. They also [27] proposed a single-radiator cassette tag for high-frequency (HF) and ultra-high-frequency (UHF) dual-frequency RFID positioning. In addition, these authors proposed some new design of miniature antennas which are suitable for UWB devices [28] [29] [30].

Sadeghzadeh et al. [31] proposed a novel miniature ultra-wideband antenna with better radiation characteristics. Limiti et al. [32] proposed a thin light antenna based on a periodic array with complementary artificial magnetic conductor metamaterial structures. Alibakhshi-Kenari et al. [33] analyzed the matching and radiation characteristics of subwavelength resonant patch antennas filled with different metamaterial blocks. Lee et al. [34] integrated a small resonant antenna with a portable RF module using a composite right/left hand transmission line.

The focus of this paper is on passive RFID that works at ultra-high frequency (UHF) band (865-928 MHz), which can identify an object up to a distance of 7 meters [7]. RFID tag uses globally unique ID for the recognition of object, which can help to solve the ambiguity problem in laser-based or visual-based approaches. As compared to visual tracking, it does not need any classification or recognition algorithms and solves the problem of visual occlusion. The laser sensor can provide the distance to the surrounding objects. But, it is difficult to distinguish the tracking object from the surrounding objects in a complex environment, which results in ambiguity of localization. We use the unique identifier of RFID to solve the ambiguity problem of laser sensors. It is well known that the phase is a periodic function of the distance (with a period of 360 degrees), which means the same phase value will repeat at the distances with an interval of the wavelength. If the radial distance that object moves is larger than a wavelength, the phase might experience multiple rotations of 360 degrees. In this case, the radial velocity computed from the phase difference is inaccurate, which leads to a large positioning error. We incorporate RFID signal strength information into the particle filter using a pre-trained sensor model to overcome this shortcoming and improve its positioning accuracy. Similar to [20], we use radial velocity matching-based approach to fuse the phase and laser sensor information. Extensive experiments (i.e., different walking velocities and multiple tag scenarios) are conducted to show the effective of our approach.

\section{System Description}

Due to the increased complexity of the indoor environment, the positioning accuracy of a single source of sensory information is not satisfactory. The laser sensor used in this paper can only obtain the location information of objects in the environment, but can not separate the target being tracked from a large number of objects in the environment. However, RFID tags have unique IDs, which can be easily used for the purpose of identification. Therefore, this paper incorporates the phase information to identify the object through velocity matching, but velocity matching needs to rely on a precise estimation of the RFID phase. The 360 degree phase rotation introduces a large amount of error to the phase velocity estimation and as a 
result leads to a poor positioning accuracy. Therefore, this paper further combines signal strength information to reduce the positioning error.

This paper focuses on localization of a dynamic object affixed with an RFID tag by the integration of RFID (i.e., RSS and phase) and laser ranging information. Therefore, the input of the system is the information collected by the two sensors (i.e., RFID system and 2D laser range finder), and the output is the position of the moving object. Specifically, we fuse RFID signal strength into a particle filter using a pre-trained sensor model. We further integrate RFID phase and laser ranging information through radial velocity matching. Fig. 1 shows an overview of our positioning system.

A person wears a tag and walks in front of the robot. Our system collects RFID (RSS and the phase) and laser ranging information. Since the location information of the tag is not available by the RFID sensor itself, we investigated the possibility of using the signal strength and phase information to track the RFID tag.

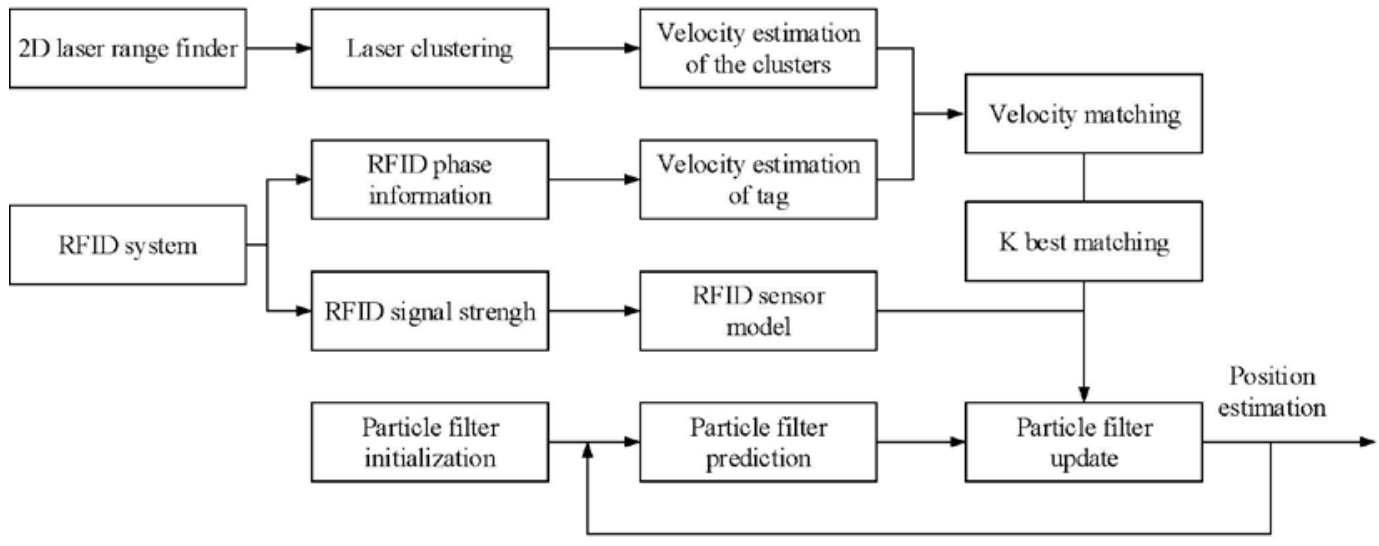

Fig. 1. System overview

Due to high resolution of the modern laser range finders, we often observe more than one laser beams reflected from a moving object. To better represent the object and compute the radial velocity, we first segment the raw laser data into clusters. Then, we calculate the laser-based radial velocity by laser clustering and phase-based radial velocity using RFID phase difference. We compute the similarity between the laser-based radial velocity and the RFID phase-based radial velocity. By sorting these similarity values, we select the $K$ clusters (i.e., objects) with the highest similarity values. These clusters will be incorporated into the particle filter for weight updates.

If the object moves too fast, the radial velocity estimated from the phase difference is not correct due to the 360 degree rotation of the phase. This will result in a large positioning error during the tracking of the object. To improve the system's positioning accuracy, we incorporate RFID signal strength using a pre-trained sensor model.

\section{Dynamic Object Tracking based on a Particle Filter}

Our goal is to determine the location $X_{t}$ of a moving object, i.e., the posterior probability $p\left(X_{t} \mid Z_{1: t}, r_{1: t}, u_{1: t}\right)$ at time $t . Z_{t}$ represents RFID measurement at time $t, u_{t}$ and $r_{t}$ represent the motion information and laser sensor measurement at time $t$. The posterior probability 
$p\left(X_{t} \mid Z_{1: t}, r_{1: t}, u_{1: t}\right)$ can be decomposed as:

$$
p\left(X_{t} \mid Z_{1: t}, r_{1: t}, u_{1: t}\right)=\eta_{t} \cdot p\left(X_{t} \mid X_{t-1}, u_{t}\right) \cdot p\left(Z_{t} \mid X_{t}\right) \cdot p\left(r_{t} \mid X_{t}, Z_{t}\right) \cdot p\left(X_{t-1} \mid Z_{1: t-1}, r_{1: t-1}, u_{1: t-1}\right)
$$

where $\eta_{t}$ is a normalizer. $p\left(X_{t} \mid X_{t-1}, u_{t}\right)$ is motion model, which predicts the location of an object $X_{t}$ at time $t$ given the motion information $u_{t}$ and the previous location $X_{t-1} \cdot p\left(Z_{t} \mid X_{t}\right)$ is RFID sensor model that represents the likelihood of obtaining a detection $Z_{t}$ given $X_{t}$. Here an RFID measurement $Z_{t}$ carries two pieces of information, i.e., $Z_{t}=\{d$,s , where $d$ indicates whether the tag is detected and sindicates signal strength when the tag is detected. $p\left(r_{t} \mid X_{t}, Z_{t}\right)$ is sensor model that incorporates the laser and RFID information using radial velocity matching. $p\left(X_{t-1} \mid Z_{1: t-1}, r_{1: t-1}, u_{1: t-1}\right)$ is status of object at time $t-1$.

\subsection{RFID Sensor Model}

The RFID sensor model represents the likelihood of getting a tag detection $Z$ at a location $(x, y)$ in antenna coordinate system. In this paper, we use a semi-autonomous learning method to establish the sensor model [13]. Several RFID tags are installed on the wall and actual locations of these tags are known. We manually control a mobile robot in the environment to collect the measurements (RFID detections and the robot's pose, which is determined using a laser-based Monte Carlo localization approach). After collecting the detections, sensor model is built through an offline process. As described before, RFID reader provides information whether the tag is detected or not (i.e., $d$ ) and the corresponding signal strength (i.e., $s$ ), therefore the probability of receiving a detection $Z$ at location $(x, y)$ can be described as:

$$
p(Z \mid(x, y))=p(d \mid(x, y)) p(s \mid d,(x, y))
$$

where $p(d \mid(x, y))$ indicates the probability of detecting a tag at $(x, y)$ and $p(s \mid d,(x, y))$ represents the likelihood of receiving a signal strength $s$ at $(x, y)$ when the tag is detected. This paper uses a two-dimensional grid to represent the sensor model. If a tag is detected in a grid $(x, y)$, we define it as a positive detection, otherwise a negative detection. $p(d \mid(x, y))$ can be obtained using the following formula:

$$
p(d \mid(x, y))=\frac{m_{(x, y)}^{+}}{m_{(x, y)}^{+}+m_{(x, y)}^{-}}
$$

where $m_{(x, y)}^{+}$is the number of positive detections in grid $(x, y)$ and $m_{(x, y)}^{-}$is the number of negative detections in grid $(x, y)$. The signal strength at a given location is assumed to be a Gaussian with mean $u_{(x, y)}$ and variance $\sigma_{(x, y)}$. The likelihood of receiving a signal strength $s$ at this location can be obtained as:

$$
p(s \mid d,(x, y))=\frac{1}{\sqrt{2 \pi} \sigma_{(x, y)}} \cdot \exp \left(-\frac{\left(s-u_{(x, y)}\right)^{2}}{2 \sigma_{(x, y)}^{2}}\right)
$$

\subsection{Laser Clustering and Radial Velocity Estimation}

In order to accurately localize an object, we cluster the data returned by the laser sensor. We first create groups from the laser ranging data and then split the group into clusters [35]. After grouping and splitting, we set the guide line as diameter to create a circle as a representation of the cluster. The clustering result at time $t$ is described as: 


$$
C_{t}=\left\{C_{t}^{(i)}\right\}_{i=1}^{N_{t}}=\left\{r_{t}^{(i)}, \bar{X}_{t}^{(i)}, \bar{y}_{t}^{(i)}\right\}_{i=1}^{N_{t}}
$$

where $r_{t}^{(i)}$ is radius of the $i$ th cluster, $\left(x_{t}^{-(i)}, y_{t}^{(i)}\right)$ is center of the $i$ th cluster, and $N_{t}$ is the number of clusters at time $t$. Since the object to be extracted in this paper is a human, the radius of the object should be limited with a certain range $R_{\max }$. After this step, discrete laser ranging points are clustered into many clusters.

For each cluster $C_{t}^{(i)}$ at time $t$, we find the closest cluster $C_{t-1}^{(\hat{j})}$ at time $t-1$. $\left(C_{t}^{(i)}, C_{t-1}^{(\hat{j})}\right)$ could be considered as the same object. $\hat{j}$ is given by:

$$
\hat{j}=\arg \min _{j} \sqrt{\left(\bar{x}_{t}^{-(i)}-\bar{x}_{t-1}^{(j)}\right)^{2}+\left(\bar{y}_{t}^{(i)}-\bar{y}_{t-1}^{(j)}\right)^{2}}
$$

where $1 \leq j \leq N_{t-1}$. The radial velocity of cluster $i$ at time $t$ is computed as:

$$
v_{t}^{(i)}=\frac{\sqrt{\left(\bar{x}_{t}^{(i)}\right)^{2}+\left(\bar{y}_{t}^{(i)}\right)^{2}}-\sqrt{\left(\bar{x}_{t-1}^{(\hat{j})}\right)^{2}+\left(\bar{y}_{t-1}^{(\hat{j})}\right)^{2}}}{\Delta t}
$$

\subsection{RFID Phase Radial Velocity Estimation based on Phase Difference}

The RFID reader activates the circuitry in the RFID tag by transmitting radio waves. After harvesting enough energy, RFID tag backscatters the radio signals. The total phase rotation of a tag is expressed as:

$$
\theta=2 \pi \cdot \frac{2 R}{\lambda}+\theta_{T}+\theta_{T A G}+\theta_{R}
$$

where $\lambda$ is the wavelength, $2 R$ is the total distance the signal travels, $\theta_{T}$ is the phase rotation of reader's transmission circuit, $\theta_{\text {TAG }}$ is the phase rotation caused by the tag, and $\theta_{R}$ is the phase rotation introduced by the receiver. Based on the phase values at two adjacent timestamps, we can calculate the radial velocity of the tag. Let $\theta_{t-1}$ and $\theta_{t}$ denote the phase readings at time $t-1$ and $t$, the radial distance moved by the tag during time $\Delta t$ can be expressed as:

$$
d_{r}=\frac{1}{2}\left(\frac{\theta_{t}-\theta_{t-1}}{2^{12}}\right) \cdot \lambda
$$

where $d_{r}<\frac{\lambda}{2}$, therefore radial velocity of the tag $v_{t}^{(r)}$ can be computed as:

$$
v_{t}^{(r)}=\frac{d_{r}}{\Delta t}
$$

\subsection{Radial Velocity Matching between RFID and Laser Clusters}

Although the laser clustering presented in section 3.2 can be used to obtain location of the object, it has an ambiguity problem to identify the object, as there may be more than one clusters extracted from laser ranging data, therefore we need to identify the respective cluster from a large number of clusters. RFID provides a solution to this issue due to its unique identification. The phase information from RFID can be used to estimate radial velocity of the moving object. The cluster with the closest radial velocity to RFID phase-based radial velocity is regarded as the object to be tracked. In this paper, we use $\operatorname{sim}\left(v_{t}^{(i)}, v_{t}^{(r)}\right)$ to measure the 
similarity between the two estimated velocities (i.e., phase radial velocity $v_{t}^{(r)}$ and laser radial velocity $\left.v_{t}^{(i)}\right)$ :

$$
\operatorname{sim}\left(v_{t}^{(i)}, v_{t}^{(r)}\right)=1-\frac{\left|v_{t}^{(i)}-v_{t}^{(r)}\right|}{\left|v_{t}^{(i)}\right|+\left|v_{t}^{(r)}\right|}
$$

a larger $\operatorname{sim}\left(v_{t}^{(i)}, v_{t}^{(r)}\right)$ value means both velocities are very close at time $t$ and it is more likely that the corresponding cluster is the object we want to localize. To improve the localization accuracy, we select the $K$ clusters with the highest similarities score (i.e., $\left.C_{t}^{\pi(1)}, \ldots, C_{t}^{\pi(K)}\right)$ to fuse with the particle filtering.

\subsection{Particle Filtering for Sensor Fusion}

Due to the non-linear and non-Gaussian characteristics, we choose the partilce filter as the implementation of the Bayesian interface. The particle filtering uses randomly sampled particles to represent the probability density distribution. The position of a moving object is represented by a set of weighted particles, i.e., $X_{t}=\left\{X_{t}^{[n]}, w_{t}^{[n]}\right\}_{n=1}^{N}$, where $N$ is the number of particles, $X_{t}^{[n]}=\left\{X_{t}^{[n]}, y_{t}^{[n]}\right\}$ is the particle's two-dimensional coordinates and $w_{t}^{[n]}$ is the weight of the particle. The particle filtering performs prediction, update, and resampling based on the measurements obtained by the RFID sensor and laser sensor, which will be described in the rest of this section.

\subsubsection{Prediction Stage}

Due to uncertainty of moving direction of the object, this paper uses the Gaussian function as prediction models. We predict the current state of a particle $X_{t}^{[n]}$ based on the previous state $X_{t-1}^{[n]}$ corrupted with a Gaussian noise:

$$
\left\{\begin{array}{l}
x_{t}^{[n]}=x_{t-1}^{[n]}+N(0, \sigma) \\
y_{t}^{[n]}=y_{t-1}^{[n]}+N(0, \sigma)
\end{array}\right.
$$

where $\sigma$ represents the standard deviation of a Gaussian white noise. A suitable $\sigma$ has to be chosen to represent moving uncertainty of the object; otherwise, the particle filtering will face the problem of tracking failure where there is no particle sits around the true location of the object.

\subsubsection{Update Stage}

At this stage, we update the weights of particles by the sensor model, which is a combination of RFID signal strength model and radial velocity matching model presented earlier in this paper. We first update the particle weights by using the best $K$ matched laser clusters obtained from the radial velocity matching:

$$
\begin{aligned}
& w_{t}^{[n]}=\eta_{t} \cdot w_{t}^{[n]} \cdot \sum_{K}^{i=1} \operatorname{sim}\left(v_{t}^{\pi(i)}, v_{t}^{(r)}\right) \exp \left(-\frac{1}{2} d^{2}\left(X_{t}^{[n]}, C_{t}^{\pi(i)}\right)\right) \\
& d^{2}\left(X_{t}^{[n]}, C_{t}^{\pi(i)}\right)=\frac{\left(x_{t}^{[n]}-\bar{x}_{t}^{\pi(i)}\right)^{2}}{\rho}+\frac{\left(y_{t}^{[n]}-\bar{y}_{t}^{\pi(i)}\right)^{2}}{\rho}
\end{aligned}
$$

where $\rho$ denotes a translational coefficient which affects the weight distribution. To achieve better positioning result, the sensor model built in section 3.1 is used to further constrain the weights of the particle filter: 


$$
w_{t}^{[n]}=\eta \cdot w_{t-1}^{[n]} \cdot p\left(Z_{t} \mid X_{t}^{[n]}\right)
$$

After updating weights of the particles, resampling is performed by duplicating the particles with high weights and removing particles with small weights. The position of the moving object is determined as a weighted mean among all particles after resampling.

\section{Experimental Results}

\subsection{Experimental Setup}

We verified the proposed approach on a SCITOS G5 service robot from Metralabs as shown in Fig. 2. A laser sensor (SICK S300) is mounted on the robot. The laser sensor provides a measuring distance up to 20 meters with a resolution of $0.5^{\circ}$ and measuring angle of $270^{\circ}$. A UHF RFID reader (Impinj Speedway Revolution R420) is additionally mounted on the robot. The reading distance of the reader is up to 7 meters. Two circularly polarized antennas (Lairs Technologies SS8688P) are connected to the reader with a height of 0.8 meters and an angle of $\pm 45^{\circ}$ to the forward moving direction of the robot. To train the RFID sensor model, 65 RFID tags (Alien Squiggle Wet Inlay) were installed on the walls with a height similar to the antenna. The physical locations of these tags are known. The sampling rate of the reader is set to be 0.4 seconds per sample.

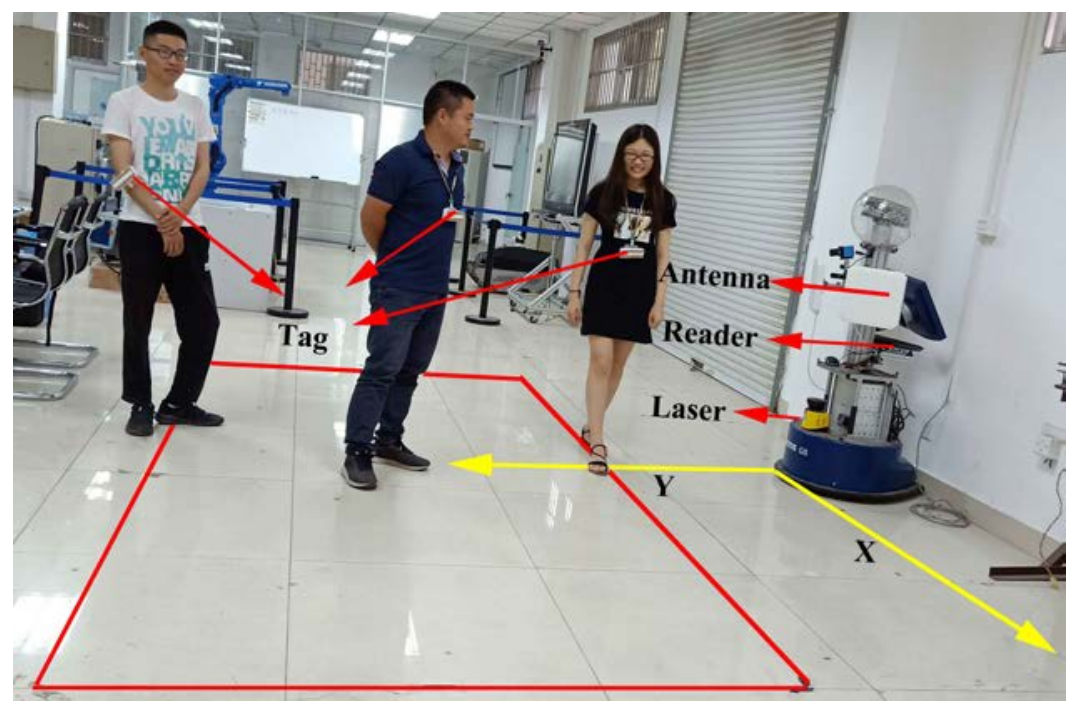

Fig. 2. A snapshot of the experiment

The robot was controlled to move on different paths in the corridor at a moving velocity of $0.2 \mathrm{~m} / \mathrm{s}$. The experiment lasted for 9 hours and the robot moved for a distance of 6500 meters. An example of the sensor model is shown in Fig. 3. Fig. 3(a), Fig. 3(b), and Fig. 3(c) give the sensor model for different signal strengths. By comparing the different signal strength models from Fig. 3, we can know that the lower the signal strength, the larger the uncertainty of the object position. Fig. 3(d) gives the average signal strength. It can be clearly seen from Fig. 3(d) that the closer the antenna, the stronger the signal strength. To evaluate the tracking accuracy, we placed the robot at a fixed position, as shown in Fig. 2. A person carried an RFID tag and walked along a rectangle with a size of $4 \mathrm{~m} \times 2 \mathrm{~m}$ at a velocity of approx $0.4 \mathrm{~m} / \mathrm{s}$. In total, 5 rounds of measurements were recorded. 


\subsection{Analysis of Experimental Results}

We first analyzed the positioning accuracy under different types of tags and different number of antennas, and then examined the positioning accuracy with RSS alone, radial velocity matching alone, and a combination of RSS and radial velocity matching. Afterwards, the impact of different parameters of our approach are analyzed, for example the number of particles $N$, the values of $K$, prediction parameter $\sigma$, update parameter $\rho$, different types of tags, and different walking velocities.

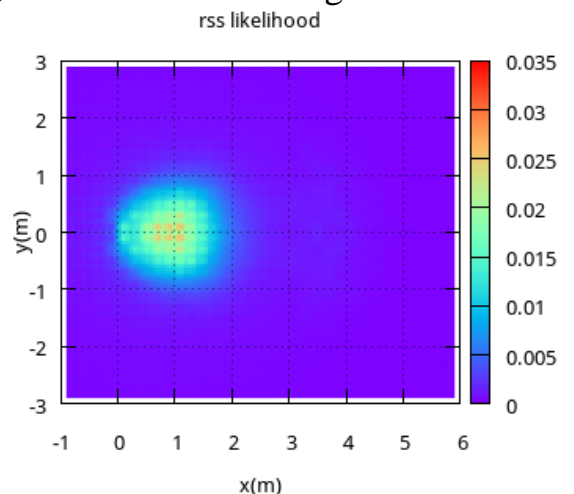

(a)

rss likelihood

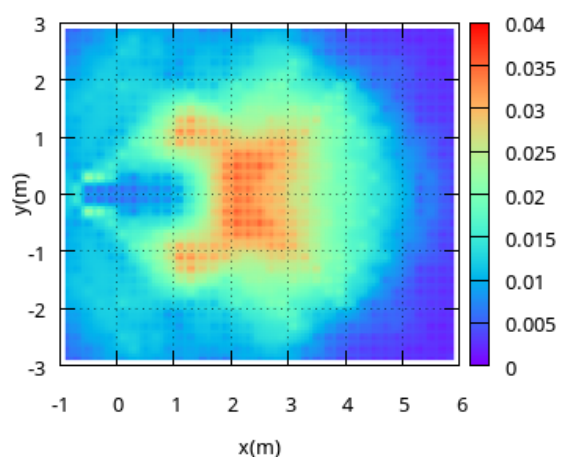

(c)



(b)



(d)

Fig. 3. Illustration of RFID sensor models. (a) Sensor model with an RSS of -48 dBm; (b) Sensor model with an RSS of -55 dBm; (c) Sensor model with an RSS of -65 dBm; (d) Mean received signal strength.

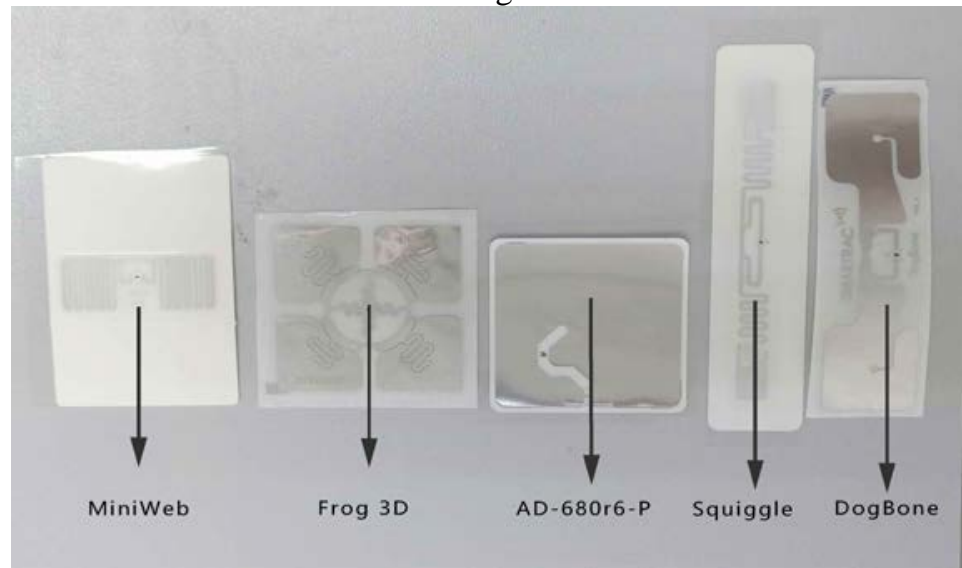

Fig. 4. Different types of tags used to test the positioning accuracy 
Table 1. Effects of different types of tags on the positioning error in meters under different approaches

\begin{tabular}{|c|c|c|c|}
\hline \multirow{2}{*}{ Type of tags } & \multicolumn{3}{|c|}{ Positioning approach } \\
\cline { 2 - 4 } & RSS[13] & $\begin{array}{c}\text { Radial velocity } \\
\text { matching[20] }\end{array}$ & Ours \\
\hline MiniWeb & 0.95 & 0.41 & 0.28 \\
\hline Frog 3D & 0.92 & 0.44 & 0.31 \\
\hline AD-680r6-P & 1.16 & 0.40 & 0.33 \\
\hline Squiggle & 0.77 & 0.41 & 0.25 \\
\hline DogBone & 0.89 & 0.43 & 0.32 \\
\hline
\end{tabular}

\subsubsection{Influence of Different Types of Tags on Positioning Accuracy}

This section discusses the impact of different types of tags on the positioning result. As can be seen from Fig. 4, we used five different types of tags (namely Smartrac MiniWeb, Smartrac Frog 3D, Avery Dennison AD-680r6-P, Alien Squiggle, and Smartrac DogBone), to test the positioning accuracy. As shown in Fig. 5 and Table 1, with RSS alone, the Squiggle tag gives a relatively good positioning result $(0.77 \mathrm{~m})$. The reason can be explained as follows: owing to different designs, different types of tags may have different propagation characteristics. Therefore, the sensor model built based on the Squiggle tag is not suitable for other types of tags. We compared the method to different references in the literature in Table 1 and added a paragraph to discuss about the results. To show the effectiveness of our approach, we compared our combined approach with two approaches in the literature, namely RSS-based approach [13] and radial velocity matching-based approach [20]. The combined approach shows better positioning accuracy for all types of tags, for example the Squiggle tag reaches a positioning accuracy of $0.25 \mathrm{~m}$, which is an improvement of $67.53 \%$ and $39.02 \%$ when compared to signal strength-based approach $(0.77 \mathrm{~m})$ in and velocity matching-based approach $(0.41 \mathrm{~m})$. To summarize, our approach can significantly improve the positioning accuracy regrading to different types of tags used when compared to RSS-based approach [13] and radial velocity matching-based approach [20].

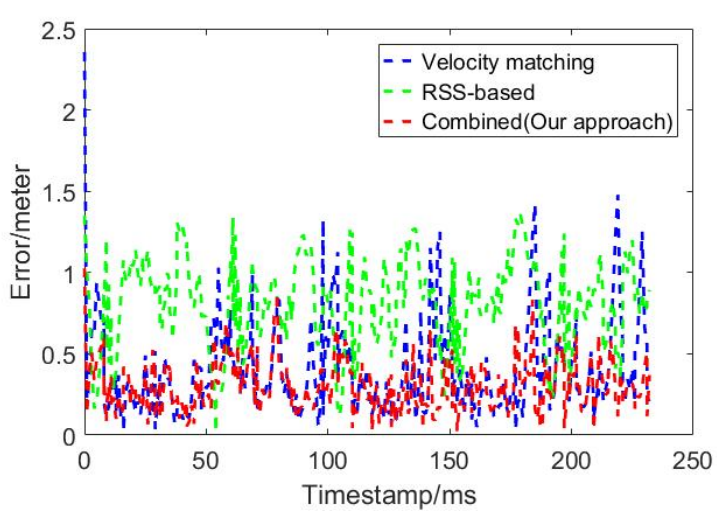

(a)



(b) 


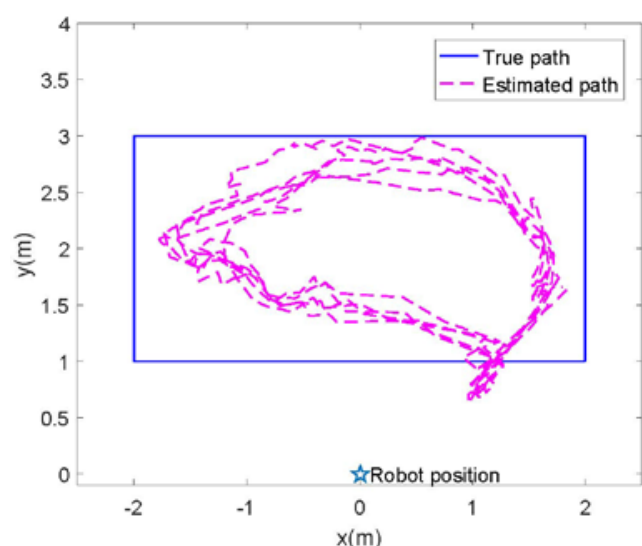

(c)

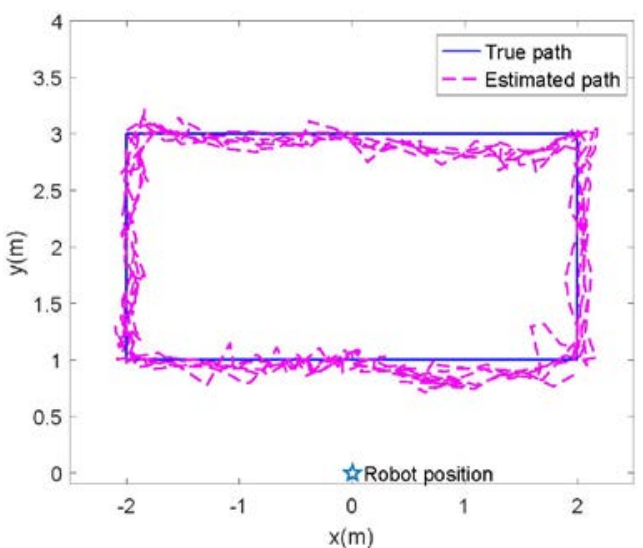

(d)

Fig. 5. Evaluation of positioning accuracy under different approaches using Squiggle tag. (a) Localization error at different timestamps using different approaches; (b) Estimated track using radial velocity matching-based approach; (c) Estimated track using RSS-based approach; (d) Estimated track of our approach

\subsubsection{Influence of Different Number of Antennas Used}

The number of antennas affects the coverage of the RFID reader, which leads to different positioning accuracy. In this section, we examined the influence of using different numbers of antennas on positioning results. Table 2 gives a comparison of the positioning results. As can be seen from Table 2, the antenna configuration has a significant influence on the positioning results. The positioning error become worse with only one antenna. Owing to the large coverage of two antennas, we achieved better positioning accuracy. By using two antennas, we obtained a positioning accuracy of $0.25 \mathrm{~m}$, which is an improvement of $62.12 \%$ and $56.90 \%$ when compared to the independent use of left antenna (i.e., $0.66 \mathrm{~m}$ ) and right antenna i.e., $0.58 \mathrm{~m}$ ), respectively. Therefore, we choose two antennas for the rest of experiments.

In this paper, we choose $K$ best matches to further restrict the weights of the particles, therefore the choice of $K$ affects the updating of the particle filter and positioning accuracy. The number of particles $N$ also affects the positioning result and the average updating time of particles. This section evaluated the effect of different $K$ and $N$ on the experimental results. The results are listed in Table 3 and Table 4. As it can be seen in Table 3, with a small $K \leq 4$, the positioning error is large, due to the small number of matched clusters used for positioning. The positioning accuracy increases with the increase of $K$. When $K \geq 9$, the positioning error basically remains stable. As it can be seen from Table 4 that a small $N \leq 10$ results in a large positioning error ( 0.483 meters). When the number of particles $N \geq 100$, we obtain almost the same positioning accuracy ( 0.255 meters). Running the algorithm with a large number of particles $N$ obviously takes more time. Considering the accuracy and running time of our approach, we choose $K=9$ and $N=100$ throughout this paper.

Table 2. Positioning error under the impact of different number of antennas

\begin{tabular}{|c|c|c|c|}
\hline $\begin{array}{c}\text { Antenna } \\
\text { configuration }\end{array}$ & Only right antenna & Only left antenna & Two antennas \\
\hline Accuracy (m) & 0.58 & 0.66 & 0.25 \\
\hline
\end{tabular}




\subsubsection{Impact of $K$ and $N$ on the Positioning Accuracy}

Table 3. Influence of different $K$ on positioning error

\begin{tabular}{|c|c|c|c|c|c|}
\hline$K$ & 1 & 4 & 9 & 13 & 100 \\
\hline Accuracy(m) & 0.357 & 0.325 & 0.255 & 0.251 & 0.252 \\
\hline
\end{tabular}

Table 4. Influence of different number of particles $N$ on the positioning error and updating time (ms)

\begin{tabular}{|c|c|c|c|c|}
\hline$N$ & 10 & 50 & 100 & 200 \\
\hline Accuracy (m) & 0.483 & 0.341 & 0.255 & 0.253 \\
\hline Running time (ms) & 0.030 & 0.079 & 0.137 & 0.245 \\
\hline
\end{tabular}

\subsubsection{Influence of $\sigma$ and $\rho$ on the Experimental Results}

In our approach, $\sigma$ and $\rho$ are two important parameters of the particle filtering. $\sigma$ controls the distribution of particles in the prediction stage and $\rho$ adjusts weights of the particles in the update stage. In this section, we analyzed the effects of $\sigma$ and $\rho$ on the positioning results. We set $\sigma$ to the following values $\{0.2,0.7,1.0,2.0\}$ and $\rho$ as follows $\{0.001,0.05,0.5,1.0\}$. The results are shown in Fig. 6. It can be seen from Fig. 6 that $\sigma$ and $\rho$ have high influence on the positioning accuracy.With $\sigma \leq 0.2$, the particle filtering can not catch the movement of the object and results in a larger positioning error. The positioning accuracy increases with the increase of $\sigma$. With $\sigma \geq 0.2$, the particle filter can not converge to the true position of object due to the large noise added in the prediction stage. With $\sigma=1.0$, the degree of particle dispersion is more moderate and gives the best positioning accuracy. $\rho$ plays an important role in the update stage and also has an imapct on the positioning error. As shown in Fig. 6, $\rho=0.001$ gives a positioning accuracy of $0.43 \mathrm{~m}$, and $\rho=1.0$ gives a positioning accuracy of $0.37 \mathrm{~m}$. Choosing too large or too small value of $\rho$ will produce a large positioning error. $\rho=0.05$ gives the best positioning accuracy of $0.25 \mathrm{~m}$, which is an improvement of $67.53 \%$ and $39.02 \%$ when compared to the settings of $\rho=0.001$ (i.e., $0.43 \mathrm{~m}$ ) and $\rho=1.0$ (i..e, $0.37 \mathrm{~m}$ ), respectively. The positioning accuracy of $\rho=0.05$ increases from 0.37 to $0.25 \mathrm{~m}$, an increases of 39.02\% compared with $\rho=1.0$. Fig. 6(b), 6(c), and 6(d) show a comparision of the trajectories under different $\rho$.

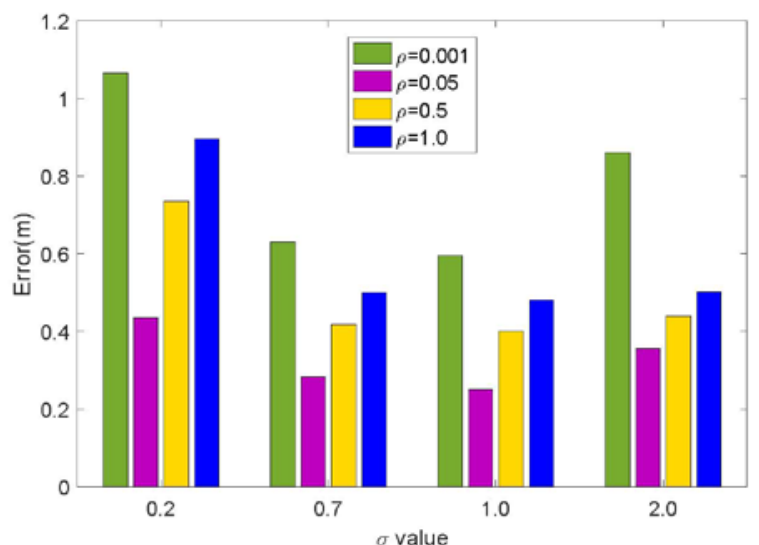

(a)

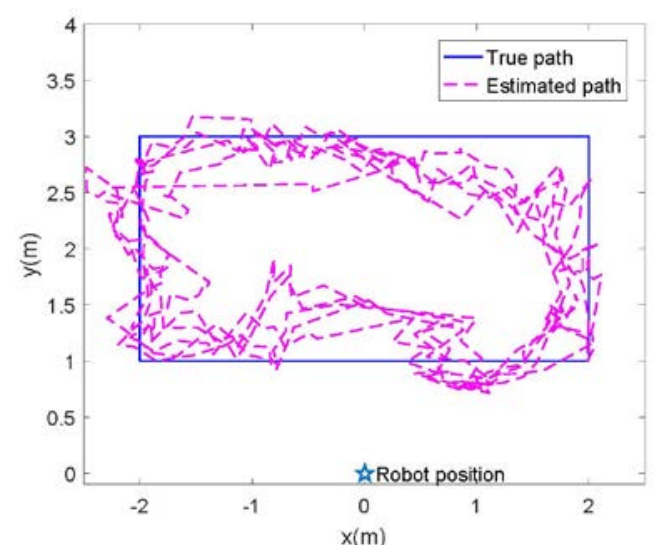

(b) 


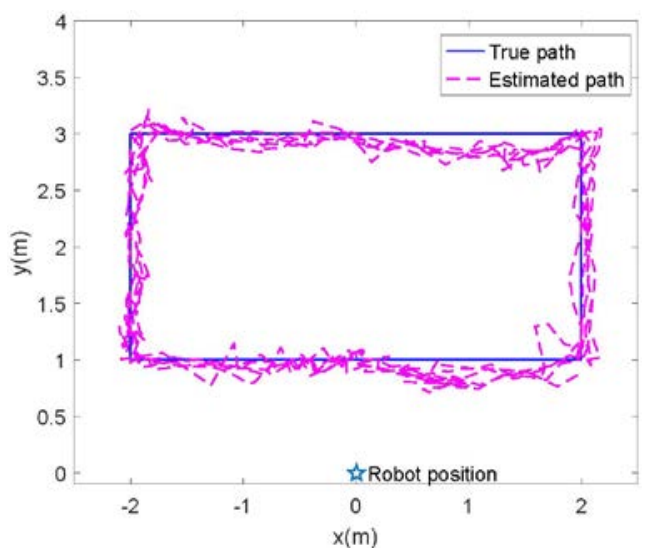

(c)

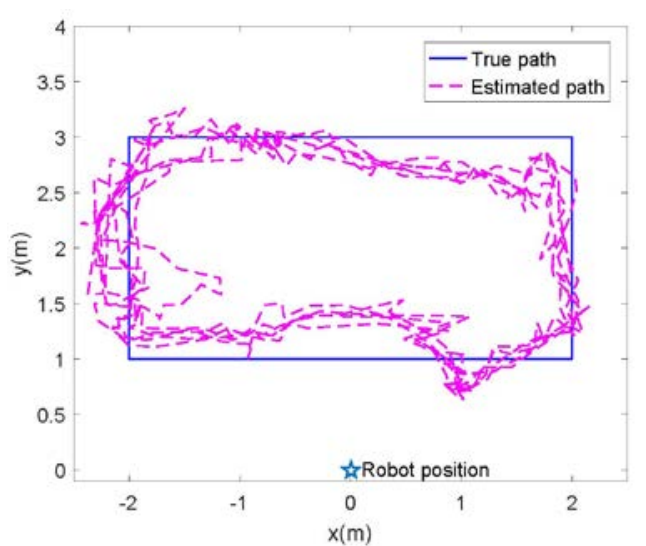

(d)

Fig. 6. Influence of different $\sigma$ and $\rho$ on the positioning error. (a) Positioning error with different $\sigma$ and $\rho$; (b) Estimated track with $\rho=0.001$; (c) Estimated track with $\rho=0.05$; (d) Estimated track with $\rho=1.0$

\subsubsection{Influence of Different Walking Velocities on Positioning Accuracy}

In this section, we evaluated the positioning error with the influence of different moving velocities, namely $0.02 \mathrm{~m} / \mathrm{s}, 0.2 \mathrm{~m} / \mathrm{s}, 0.4 \mathrm{~m} / \mathrm{s}$ and $0.6 \mathrm{~m} / \mathrm{s}$. A comparison of the positioning accuracy is shown in the Table 5 . As shown in this table, when the moving velocity of the object is small $(0.02 \mathrm{~m} / \mathrm{s})$, the radial velocity matching can not effectively distinguish the moving objects from the stationary object, resulting in a large positioning error $(0.75 \mathrm{~m})$. When the moving speed is higher than $0.6 \mathrm{~m} / \mathrm{s}$, the radial distance of the dynamic target moving at the adjacent time is larger than half a wavelength, resulting in a large radial velocity estimation error and a poor positioning accuracy. We obtain the best positioning accuracy (i.e., $0.25 \mathrm{~m}$ ) with a velocity of $0.4 \mathrm{~m} / \mathrm{s}$, which is an improvement of $66.67 \%$ and $56.90 \%$ when compared to a velocity of $0.02 \mathrm{~m} / \mathrm{s}$ (accuracy of $0.75 \mathrm{~m}$ ) and $0.6 \mathrm{~m} / \mathrm{s}$ (accuracy of $0.44 \mathrm{~m}$ ), respectively.

Table 5. Effects of different moving velocities on the positioning error

\begin{tabular}{|c|c|c|c|c|}
\hline Moving Velocity (m/s) & 0.02 & 0.2 & 0.4 & 0.6 \\
\hline Accuracy (m) & 0.75 & 0.29 & 0.25 & 0.44 \\
\hline
\end{tabular}

\section{Conclusion}

This paper proposed a method to fuse RFID and laser ranging information for moving object tracking. We use a particle filter to fuse the signal strength by an RFID sensor model and laser ranging information through radial velocity matching. The proposed approach takes advantages of RFID and a laser sensor and provides a better positioning accuracy as compared to the state-of-the-art approaches. We validated the proposed method on a service robot. Experimental results proved that we can achieve a positioning accuracy of $0.25 \mathrm{~m}$ by integrating RFID and laser ranging information, which is an improvement of $67.53 \%$ and $39.02 \%$ as compared to RSS-based approach $(0.77 \mathrm{~m})$ and radial velocity matching-based approach $(0.41 \mathrm{~m})$ respectively. The proposed approach provides a solution to precisely track dynamic objects by combining the laser range and RFID information. The proposed approach has potential applications for human machine interaction and object tracking in many fields 
for example supermarkets, libraries, shopping malls, and exhibitions. In our current work, the prediction of the object is done by a random walk, therefore a future research direction is to incorporate the moving direction and velocity of the object to reduce the positioning error.

\section{References}

[1] S. N. He and S-H. G. Chan, "Wi-Fi fingerprint-based indoor positioning: Recent advances and comparisons,” IEEE Communications Surveys Tutorials, vol. 18, no. 1, pp. 466-490, 2016. Article (CrossRef Link)

[2] A. Yassin, Y. Nasser, M. Awad, A. Al-Dubai, R. Liu, C. Yuen, R. Raulefs, "Recent Advances in Indoor Localization: A Survey on Theoretical Approaches and Applications," IEEE Communications Surveys Tutorials, vol. 19, no. 2, pp. 1327-1346 , 2017. Article (CrossRef Link)

[3] R. Liu and C. Yuen and T. N. Do and U. X. Tan, "Fusing Similarity-Based Sequence and Dead Reckoning for Indoor Positioning Without Training,” IEEE Sensors, vol. 17, no. 13, pp. 4197-4207, July, 2017. Article (CrossRef Link)

[4] R. Liu and C. Yuen and T. N. Do and D. W. Jiao and X. Liu and U-X. Tan, "Cooperative Relative Positioning of Mobile Users by Fusing IMU Inertial and UWB Ranging Information," in Proc. of the 2017 IEEE International Conference on Robotics and Automation (ICRA 2017), PP. 5623-5629, 2017. Article (CrossRef Link)

[5] R. Liu, C. Yuen, T. N. Do, Y. Jiang, X. Liu, and U-X. Tan "Indoor positioning using similarity-based sequence and dead reckoning without training," in Proc. of the 19th IEEE International workshop on Signal Processing advances in Wireless Communications (SPAWC 2017), PP. 1-5, 2017. Article (CrossRrf Link)

[6] H. Ma and K. Wang, "Fusion of RSS and Phase Shift Using the KalmanFilter for RFID Tracking,” IEEE Sensors Journal, vol. 17, no. 11, pp. 3551-3558, Apr, 2017.

Article (CrossRef Link)

[7] F. Seco and A. R. Jime 'nez, "Smartphone-Based Cooperative Indoor Localization with RFID Technology,” Sensors, vol. 18, no. 1, pp. 266-266, Jan, 2018. Article (CrossRef Link)

[8] R. Liu and A. Zell, "On tracking dynamic objects with long range passive UHF RFID using a mobile robot," International Journal of Distributed Sensor Networks, Art. no. 781380, Jan. 2015. Article (CrossRef Link)

[9] R. Liu, A. Koch, and A. Zell, "Mapping UHF RFID Tags with a Mobile Robot using a 3D Sensor Model," in Proc. of the 2013 IEEE/RSJ International Conference on Intelligent Robots and Systems (IROS 2013), pp. 1589-1594, 2013. Article (CrossRef Link)

[10] L. Xie, Q. Li, and C. Wang, "Exploring the Gap between Ideal and Reality: An Experimental Study on Continuous Scanning with Mobile Reader in RFID Systems," IEEE Transactions on Mobile Computing, vol. 14, no. 11, pp. 2272-2285, Nov, 2015. Article (CrossRef Link)

[11] G. Cicirelli, A. Milella, and D. D. Paola, "Supervised learning of RFID sensor model using a mobile robot," in Proc. of the IEEE International Conference on Rfid-Technologies and Applications, pp. 32-36, 2011. Article (CrossRef Link)

[12] P. Vorst and A. Zell, "Semi-autonomous learning of an RFID sensor model for mobile robot self-localization," in Proc. of the European Robotics Symposium, pp. 273-282, 2008. Article (CrossRef Link)

[13] D. Joho, C. Plagemann and W. Burgard, "Modeling RFID signal strength and tag detection for localization and mapping," in Proc. of the 2009 IEEE International Conference on Robotics and Automation (ICRA 2009), pp. 1213-1218, 2009. Article (CrossRef Link)

[14] J. Hightower, R. Want, and G. Borriello, "Spot ON: An indoor 3D location sensing technology based on RF signal strength,” UW CSE, Washington USA, Tech. Rep. 2000-02-02, Feb. 18, 2000. Article (CrossRef Link)

[15] X. Wu, F. M. Deng and Z. B. Chen, "RFID 3D-LANDMARC Localization Algorithm Based on Quantum Particle Swarm Optimization,” Electronics, vol. 7, no. 2, pp. 19, 2018. Article (CrossRef Link) 
[16] A. P. Sample, C. Macomber, L. T. Jiang, and J. R. Smith, "Optical localization of passive UHF RFID tags with integrated LEDs,” in Proc. of the IEEE International Conference on RFID, pp. 116-123, 2016. Article (CrossRef Link)

[17] K. Rohweder and P. Vorst and A. Zell, "Improved Mapping of RFID Tags by Fusion with Spatial Structure,” in Proc. of the 4th European Conf. on Mobile Robots (ECMR 2009), pp. 247-252, 2009. Article (CrossRef Link)

[18] T. Deyle, M. S. Reynolds, and C. C. Kemp, "Finding and Navigating to Household Objects with UHF RFID Tags by Optimizing RF Signal Strength,” in Proc. of the 2014 IEEE/RSJ International Conference on Intelligent Robots and Systems (IROS 2014), pp. 2457-2586, 2014. Article (CrossRef Link)

[19] R. Liu, G. Huskic, and A. Zell, "Dynamic objects tracking with a mobile robot using passive UHF RFID tags," in Proc. of the 2014 IEEE/RSJ International Conference on Intelligent Robots and Systems (IROS 2014), pp. 4247-4252, 2014. Article (CrossRef Link)

[20] Y. L. Fu, C. L. Wang, R. Liu, G. L. Liang, H. Zhang and S. U. Rehman, "Moving Object Localization Based on UHF RFID Phase and Laser Clustering,” Sensors, vol. 18, no. 3, pp. 825, Mar, 2018. Article (CrossRef Link)

[21] M. Scherhaufl, M. Pichler, E. Schimback, D. J. Muller, and A. Ziroff, "Indoor Localization of Passive UHF RFID Tags Based on Phase-of-Arrival Evaluation,” IEEE Transactions on Microwave Theory \& Techniques, vol. 61, no. 12, pp. 4724-4729, Dec, 2013.

[22] Z. N. Chen, X. Qing, “Antennas for RFID applications,” in Proc. of International Workshop on Antenna Technology, pp. 273-282, 2010. Article (CrossRefLink)

[23] Z. N. Chen, X. Qing, H. L. Chung, “A Universal UHF RFID Reader Antenna,” IEEE Transactions on Microwave Theory and Techniques, vol. 57, no. 5, pp. 1275-1282, 2009.

Article (CrossRefLink)

[24] X. Qing, C. K. Goh, Z. N. Chen, “Segmented loop antenna for UHF near-field RFID applications,” Electronics Letters, vol. 45, no. 17, pp. 872-873, 2009. Article (CrossRefLink)

[25] X. Qing , Z. N. Chen, "Characteristics of a Metal-Backed Loop Antenna and its Application to a High-Frequency RFID Smart Shelf,” IEEE Antennas and Propagation Magazine, vol. 51, no. 2, pp. 26-38, 2009. Article (CrossRefLink)

[26] M. Alibakhshi-Kenari, M. Naser-Moghadasi, R. Sadeghzadeh, “The resonating MTM-based miniaturized antennas for wide-band RF-microwave systems," Microwave and Optical Technology Letters, vol. 57, no. 10, pp. 2339-2344, 2015. Article (CrossRefLink)

[27] M. Alibakhshi-Kenari, M. Naser-Moghadasi, R. Ali Sadeghzadeh, B. S. Virdee and E. Limiti, "Dual-band rfid tag antenna based on the hilbert-curve fractal for hf and uhf applications," IET Circuits, Devices \& Systems, vol. 10, no. 2, pp. 140 -146, 2016. Article (CrossRefLink)

[28] M. Alibakhshi-Kenari, M. Naser-Moghadasi, "Novel UWB miniaturized integrated antenna based on CRLH metamaterial transmission lines,” AEU - International Journal of Electronics and Communications, vol. 69, no. 8, pp. 1143-1149, 2015. Article (CrossRefLink)

[29] M. Alibakhshi-Kenari, M. Movahhedi, H. Naderian, "A new miniature ultra wide band planar microstrip antenna based on the metamaterial transmission line," in Proc. of the Applied Electromagnetics, IEEE, 2012. Article (CrossRefLink)

[30] M. Alibakhshi-Kenari, E. Limiti, M. Naser-Moghadasi, “A New Wideband Planar Antenna with Band-Notch Functionality at GPS, Bluetooth and WiFi Bands for Integration in Portable Wireless Systems," AEU - International Journal of Electronics and Communications, vol.72, pp. 79-85, 2017. Article (CrossRefLink)

[31] R. A. Sadeghzadeh, M. Alibakhshi-Kenari, M. Naser-Moghadasi, "UWB antenna based on SCRLH-TLs for portable wireless devices,” Microwave and Optical Technology Letters, vol. 58, no. 1, pp. 69-71, 2016. Article (CrossRefLink) 
[32] E. Limiti, M. Alibakhshi-Kenari, "Periodic array of complementary artificial magnetic conductor metamaterials-based multiband antennas for broadband wireless transceivers," IET Microwaves, Antennas \& Propagation, vol. 10, no. 15, pp. 1682-1691, 2016. Article (CrossRefLink)

[33] A. Alu, F. Bilotti, N. Engheta , "Subwavelength, Compact, Resonant Patch Antennas Loaded With Metamaterials,” IEEE Transactions on Antennas and Propagation, vol. 55, no. 1 pp.13-25, 2007. Article (CrossRefLink)

[34] C. J. Lee, K. M. K. H. Leong, T . Itoh, “Composite right/left-handed transmission line based compact resonant antennas for RF module integration,” IEEE Transactions on Antennas and Propagation, vol. 54, no. 8, pp. 2283-2291, 2006. Article (CrossRefLink)

[35] M. Przybyla, "Detection and tracking of 2D geometric obstacles from LRF data," in Proc. of the IEEE International Workshop on Robot Motion and Control, pp. 135-141, 2017. Article (CrossRef Link)

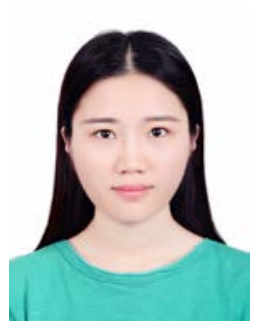

Gaoli Liang obtained her bachelor's degree from the Southwest University of Science and Technology, Mianyang, China, in 2012. She is now working as a master student at the same university. She is interested in RFID tracking and mobile robot localization.



Ran Liu received the Ph.D. degree from the University of Tuebingen, Germany, in 2014. Since then, he has been a Post-Doctoral Research Fellow at Singapore University of Technology and Design. He is an associate professor at the Southwest University of Science and Technology. He is interested in robotics, SLAM, indoor positioning, UHF RFID localization, and mapping.



Yulu Fu received the bachelor's degree from the Southwest University of Science and Technology, Mianyang, China, in 2012. She is now a master student at the same university. Her research interests include RFID positioning and object tracking. 


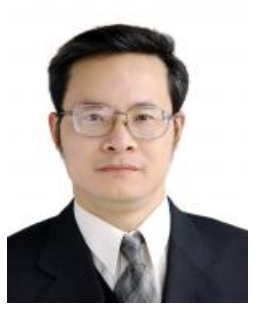

Hua Zhang is a professor at the Southwest University of Science and Technology since 2010. He received the Ph.D. degree from Chongqing University, China, in 2006. He was a visiting professor in Texas A\&M University, America from 2010 to 2011. His research interests include mobile robot and embedded technology.

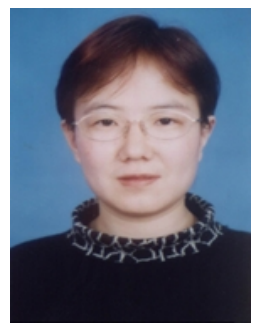

Heng Wang is a professor at the Southwest University of Science and Technology since 2011. She obtained the master degree from Chongqing University, China, in 1999. She was a visiting researcher of Tohoku University in 2004. Her research interests include mobile robot localization and mapping.

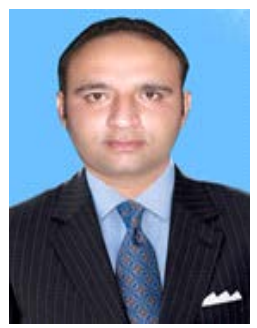

Shafiq ur Rehman is currently a PhD student in School of Information Engineering, Southwest University of Science and Technology, China. He has been a lecturer in Department of Computer Science, Lasbela University of Agriculture, Water \& Marine Sciences(LUAWMS), Pakistan since 2012. In 2005, he has completed his master in Software Engineering from COMSATS Institute of Information Technology, Pakistan. He worked for Ovex Technologies, USA as a Programmer and System Analyst from 2005 to 2008. He worked in Avenir Technologies, Pakistan as Senior Software Engineer from 2008 to 2011. His research interest includes localization and tracking of robots through RFID and other technologies.

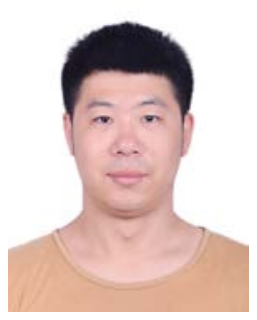

Mingming Guo obtained his bachelor's degree from the Southwest University of Science and Technology, Mianyang, China, in 2012. He is currently a master student at the same university. His research interests include manipulator motion planning and control. 